\title{
Black holes in Sol minore
}

\author{
Federico Faedo, ${ }^{a, b}$ Daniele Angelo Farotti ${ }^{a}$ and Silke Klemm ${ }^{a, b}$ \\ ${ }^{a}$ Dipartimento di Fisica, Università di Milano, \\ Via Celoria 16, I-20133 Milano, Italy \\ ${ }^{b}$ INFN, Sezione di Milano, \\ Via Celoria 16, I-20133 Milano, Italy \\ E-mail: federico.faedo@unimi.it, \\ danieleangelo.farotti@studenti.unimi.it, silke.klemm@mi.infn.it
}

ABSTRACT: We consider black holes in five-dimensional $N=2 \mathrm{U}(1)$-gauged supergravity coupled to vector multiplets, with horizons that are homogeneous but not isotropic. We write down the equations of motion for electric and magnetic ansätze, and solve them explicitely for the case of pure gauged supergravity with magnetic U(1) field strength and Sol horizon. The thermodynamics of the resulting solution, which exhibits anisotropic scaling, is discussed. If the horizon is compactified, the geometry approaches asymptotically a torus bundle over $\mathrm{AdS}_{3}$. Furthermore, we prove a no-go theorem that states the nonexistence of supersymmetric, static, Sol-invariant, electrically or magnetically charged solutions with spatial cross-sections modelled on solvegeometry. Finally, we study the attractor mechanism for extremal static non-BPS black holes with nil- or solvegeometry horizons. It turns out that there are no such attractors for purely electric field strengths, while in the magnetic case there are attractor geometries, where the values of the scalar fields on the horizon are computed by extremization of an effective potential $V_{\text {eff }}$, which contains the charges as well as the scalar potential of the gauged supergravity theory. The entropy density of the extremal black hole is then given by the value of $V_{\text {eff }}$ in the extremum.

Keywords: AdS-CFT Correspondence, Black Holes, Classical Theories of Gravity, Supergravity Models

ArXiv EPrint: 1908.07421 


\section{Contents}

1 Introduction and summary of results 1

$2 \quad N=2, D=5 \mathrm{U}(1)$-gauged supergravity 3

3 Equations of motion for electric and magnetic ansätze 4

3.1 Electric ansatz 4

3.2 Magnetic ansatz 5

4 Magnetic black hole in pure gauged supergravity $\quad 6$

5 Existence of static, Sol-invariant BPS solutions 8

$\begin{array}{llr}5.1 & \text { Electric ansatz } & 9\end{array}$

$\begin{array}{ll}5.2 \text { Magnetic ansatz } & 11\end{array}$

6 Attractor mechanism $\quad \mathbf{1 5}$

$\begin{array}{ll}\text { 6.1 Magnetic ansatz } & 16\end{array}$

$\begin{array}{lll}6.2 & \text { Electric ansatz } & 17\end{array}$

$\begin{array}{lr}\text { A Homogeneous manifolds } & 18\end{array}$

\section{Introduction and summary of results}

In the seventies of the last century Hawking proved his famous theorem $[1,2]$ on the topology of black holes, which asserts that event horizon cross sections of 4-dimensional asymptotically flat stationary black holes obeying the dominant energy condition are topologically $\mathrm{S}^{2}$. This result extends to outer apparent horizons in black hole spacetimes that are not necessarily stationary [3]. Such restrictive uniqueness theorems do not hold in higher dimensions, the most famous counterexample being the black ring of Emparan and Reall [4], with horizon topology $S^{2} \times S^{1}$. Nevertheless, Galloway and Schoen [5] were able to show that, in arbitrary dimension, cross sections of the event horizon (in the stationary case) and outer apparent horizons (in the general case) are of positive Yamabe type, i.e., admit metrics of positive scalar curvature.

Instead of increasing the number of dimensions, one can relax some of the assumptions that go into Hawking's theorem in order to have black holes with nonspherical topology. One such possibility is to add a negative cosmological constant $\Lambda$. Interpreting the term $-\Lambda g_{\mu \nu}$ as $8 \pi G$ times the energy-momentum tensor $T_{\mu \nu}$, one has obviously that $-T_{\mu \nu} \xi^{\nu}$ is past-pointing for every future-pointing causal vector $\xi^{\nu}$, and thus a violation of the dominant energy condition. Moreover, since for $\Lambda<0$ the solutions generically asymptote to anti-de Sitter (AdS) spacetime, also asymptotic flatness does not hold anymore. In this 
case, the horizon of a black hole can indeed be a compact Riemann surface $\Sigma_{g}$ of any genus $g$ [6-9]. It should be noted that, unless $g=0$, these spacetimes are asymptotically only locally AdS; their global structure is different. This is in contrast to the black rings in five dimensions, which are asymptotically Minkowski, in spite of their nontrivial horizon topology. Notice in addition that the solutions of [6-9] do not exhaust the spectrum of black holes in $\mathrm{AdS}_{4}$, since one can also have horizons that are noncompact manifolds with yet finite area (and thus finite entropy), topologically spheres with two punctures $[10,11]{ }^{1}$

In this paper, we will allow for both of the possibilities described above, i.e., we shall consider the case $D=5$ and include a negative cosmological constant. More generally, our model contains scalar fields with a potential that admits $\mathrm{AdS}_{5}$ vacua. A class of uncharged black holes in Einstein-Lambda gravity was obtained by Birmingham in [13] for arbitrary dimension $D$. These solutions have the property that the horizon is a $(D-2)$-dimensional Einstein manifold of positive, zero, or negative curvature. In our case, $D=5$, and threedimensional Einstein spaces have necessarily constant curvature, i.e., are homogeneous and isotropic. Similar to what is done in Bianchi cosmology, one can try to relax these conditions by dropping the isotropy assumption. The horizon is then a homogeneous manifold, and belongs thus to the nine 'Bianchi cosmologies', which are in correspondence with the eight Thurston model geometries, cf. appendix A for details. For two of these cases, namely Nil and Sol, the corresponding black holes in five-dimensional gravity with negative cosmological constant were constructed in [14] for the first time. Asymptotically, these solutions are neither flat nor AdS, but exhibit anisotropic scaling.

Here we go one step further with respect to [14] and add also charge. Some attempts in this direction include [15], where an intrinsically dyonic black hole with Sol horizon in Einstein-Maxwell-AdS gravity was found ${ }^{2}$ and [16], which considers different models that are not directly related to gauged supergravity theories. There are various reasons for the addition of charge. First of all, charged black holes generically have an extremal limit, and a subclass of these zero-temperature solutions might preserve some fraction of supersymmetry, which is instrumental in holographic computations of the number of microstates. Moreover, in the extremal limit we expect to find an attractor mechanism [17-21], according to which the horizon values of the scalar fields in the theory are determined by the electromagnetic charges alone, and do not depend on the asymptotic values of the moduli. In our case, the corresponding attractor geometry would be $\mathrm{AdS}_{2} \times M$, where $M$ denotes a three-dimensional homogeneous manifold. ${ }^{3}$ These issues will be addressed in the following.

We start in section 2 by setting up the gauged supergravity model that will be considered throughout the paper. In 3 we write down the equations of motion for electric and magnetic ansätze. These are then solved explicitely in section 4 for the case of pure gauged

\footnotetext{
${ }^{1}$ These solutions can be generalized to $D>4[12]$.

${ }^{2}$ Since the authors of [15] do not include a Chern-Simons term, their solution does not solve the equations of motion of pure gauged supergravity.

${ }^{3}$ Note in this context that [22] considers near-horizon geometries with various homogeneous horizons in theories containing massive vector fields, while [23] constructs solutions interpolating between some Bianchi cosmologies and Lifshitz geometries or $\mathrm{AdS}_{2} \times \mathrm{S}^{3}$. The authors of [23] do not show that these solutions can be obtained from some particular theories, but prove that the corresponding energy-momentum tensor satisfies the weak energy condition.
} 
supergravity with magnetic U(1) field strength and Sol horizon. Moreover, the thermodynamics of the resulting solution, which exhibits anisotropic scaling, is discussed. If the horizon is compactified, the geometry approaches asymptotically a torus bundle over $\mathrm{AdS}_{3}$. Section 5 is dedicated to the proof of a no-go theorem that states the nonexistence of supersymmetric, static, Sol-invariant, electrically or magnetically charged solutions with spatial cross-sections modelled on solvegeometry. Finally, in 6 we study the attractor mechanism for extremal static non-BPS black holes with nil- or solvegeometry horizons. It turns out that there are no such attractors for purely electric field strengths, while in the magnetic case there are attractor geometries, where the values of the scalar fields on the horizon are computed by extremization of an effective potential $V_{\text {eff }}$, which contains the charges as well as the scalar potential of the gauged supergravity theory. The entropy density of the extremal black hole is then given by the value of $V_{\text {eff }}$ in the extremum.

\section{$2 \quad N=2, D=5 \mathrm{U}(1)$-gauged supergravity}

We consider $N=2, D=5 \mathrm{U}(1)$-gauged supergravity coupled to $n$ abelian vector multiplets, whose bosonic field content includes the fünfbein $e_{\mu}^{a}$, the vectors $A_{\mu}^{I}$ with $I=0, \ldots, n$ and the real scalars $\phi^{i}$, where $i=1, \ldots, n$. The gauging of the $\mathrm{U}(1)$ subgroup of the $\mathrm{SU}(2) \mathrm{R}$ symmetry is achieved through the vector field $A_{\mu}=V_{I} A_{\mu}^{I}$ with coupling constant $g$, where the $V_{I}$ are constant parameters. In order to preserve supersymmetry the introduction of a scalar potential is required. The bosonic part of the Lagrangian is given by [24]

$$
e^{-1} \mathscr{L}=\frac{R}{2}-\frac{1}{2} \mathcal{G}_{i j} \partial_{\mu} \phi^{i} \partial^{\mu} \phi^{j}-\frac{1}{4} G_{I J} F_{\mu \nu}^{I} F^{J \mu \nu}+\frac{e^{-1}}{48} C_{I J K} \varepsilon^{\mu \nu \rho \sigma \tau} F_{\mu \nu}^{I} F_{\rho \sigma}^{J} A_{\tau}^{K}-g^{2} U,
$$

where $F_{\mu \nu}^{I}$ are the abelian field strength tensors. The scalar potential $U$ reads

$$
U=V_{I} V_{J}\left(\frac{9}{2} \mathcal{G}^{i j} \partial_{i} h^{I} \partial_{j} h^{J}-6 h^{I} h^{J}\right)
$$

where $\mathcal{G}^{i j}$ is the inverse of the target space metric $\mathcal{G}_{i j}, \partial_{i}$ denotes the partial derivative with respect to $\phi^{i}$ and the functions $h^{I}=h^{I}\left(\phi^{i}\right)$ satisfy the condition

$$
\mathcal{V}:=\frac{1}{6} C_{I J K} h^{I} h^{J} h^{K}=1,
$$

with $C_{I J K}$ a fully symmetric, constant and real tensor. The kinetic matrices $\mathcal{G}_{i j}$ and $G_{I J}$ are given by

$$
G_{I J}=-\left.\frac{1}{2} \frac{\partial}{\partial h^{I}} \frac{\partial}{\partial h^{J}} \log \mathcal{V}\right|_{\mathcal{V}=1}, \quad \mathcal{G}_{i j}=\left.\partial_{i} h^{I} \partial_{j} h^{J} G_{I J}\right|_{\mathcal{V}=1} .
$$

The Einstein-, Maxwell-Chern-Simons- and scalar field equations following from (2.1) are respectively

$$
\begin{aligned}
& R_{\mu \nu}=\mathcal{G}_{i j} \partial_{\mu} \phi^{i} \partial_{\nu} \phi^{j}+G_{I J}\left(F_{\mu}^{I \rho} F_{\nu \rho}^{J}-\frac{1}{6} g_{\mu \nu} F_{\rho \sigma}^{I} F^{J \rho \sigma}\right)+\frac{2}{3} g^{2} U g_{\mu \nu}, \\
& \nabla_{\lambda}\left(G_{I J} F^{J \lambda \tau}\right)+\frac{e^{-1}}{16} C_{I J K} \varepsilon^{\mu \nu \rho \sigma \tau} F_{\mu \nu}^{J} F_{\rho \sigma}^{K}=0 \\
& \nabla_{\mu}\left(\mathcal{G}_{i j} \partial^{\mu} \phi^{j}\right)-\frac{1}{2} \partial_{i} \mathcal{G}_{k j} \partial_{\mu} \phi^{k} \partial^{\mu} \phi^{j}-\frac{1}{4} \partial_{i} G_{I J} F_{\mu \nu}^{I} F^{J \mu \nu}-g^{2} \partial_{i} U=0 .
\end{aligned}
$$




\section{Equations of motion for electric and magnetic ansätze}

In order to solve the equations of motion (2.5)-(2.7) we use an ansatz inspired by [14], with homogeneous sections $\Sigma_{t, r}$ of constant $t$ and $r$. Without loss of generality, we take the line element to be

$$
d s^{2}=-V(r) d t^{2}+\frac{d r^{2}}{V(r)}+\sum_{A=1}^{3} e^{2 T_{A}(r)}\left(\theta^{A}\right)^{2},
$$

where the induced metric on $\Sigma_{t, r}$ is written in terms of $G$-invariant 1-forms $\theta^{A}$, which satisfy

$$
d \theta^{A}=\frac{1}{2} C_{B C}^{A} \theta^{B} \wedge \theta^{C}
$$

with $C_{B C}^{A}$ the structure constants of the Lie algebra of the isometry group $G$. A list of all the possible isometry groups with related structure constants and invariant 1-forms can be found in [25], while in appendix A we present those for solve- and nilgeometries along with a brief discussion of homogeneous manifolds. Henceforth we shall restrict our discussion to class A Bianchi models, which contain the most exotic cases, such as solve- and nilgeometry (cf. table 1 in appendix A).

The scalar fields are assumed to depend on the radial coordinate only,

$$
\phi^{i}=\phi^{i}(r) .
$$

\subsection{Electric ansatz}

For a purely electric ansatz the vector fields are given by

$$
A^{I}=A_{t}^{I}(r) d t
$$

and the Maxwell equations (2.6) imply

$$
F_{r t}^{I}=\partial_{r} A_{t}^{I}=e^{-\sum_{A} T_{A}} G^{I J} q_{J},
$$

where $G^{I J}$ denotes the inverse of $G_{I J}$, and the constants $q_{I}$ represent essentially the electric charge densities.

Using (3.5) and the Bianchi class A condition (A.4), the Einstein equations (2.5) boil down to

$$
\begin{aligned}
& \frac{V^{\prime \prime}}{2}+\frac{V^{\prime}}{2} \sum_{A} T_{A}^{\prime}=\frac{2}{3} e^{-2 \sum_{A} T_{A}} G^{I J} q_{I} q_{J}-\frac{2}{3} g^{2} U, \\
& \sum_{A} T_{A}^{\prime \prime}+\sum_{A}\left(T_{A}^{\prime}\right)^{2}=-\mathcal{G}_{i j} \phi^{i^{\prime}} \phi^{j^{\prime}}, \quad \sum_{B} C_{A B}^{B} T_{B}^{\prime}=0, \\
& -V^{\prime} T_{A}^{\prime}-V T_{A}^{\prime \prime}-V T_{A}^{\prime} \sum_{B} T_{B}^{\prime}+\mathcal{J}_{A}=\frac{1}{3} e^{-2 \sum_{B} T_{B}} G^{I J} q_{I} q_{J}+\frac{2}{3} g^{2} U,
\end{aligned}
$$

where we defined

$$
\mathcal{J}_{A}:=\sum_{B, C}\left[-\frac{1}{2} D_{A C}^{B}\left(D_{A B}^{C}+D_{A C}^{B}\right)+\frac{1}{4}\left(D_{B C}^{A}\right)^{2}\right],
$$


with

$$
D_{B C}^{A}:=e^{T_{A}-T_{B}-T_{C}} C_{B C}^{A} .
$$

The third equation in (3.6) is a constraint, which is trivially satisfied for all the class A Bianchi cosmologies except for solvegeometry; in this case it reduces to

$$
T_{1}^{\prime}=T_{2}^{\prime}
$$

Finally, using (3.5), the equations (2.7) for the scalars become

$$
\begin{array}{r}
V \mathcal{G}_{i j} \phi^{j^{\prime}} \sum_{A} T_{A}^{\prime}+V \frac{d \mathcal{G}_{i j}}{d r} \phi^{j^{\prime}}+V^{\prime} \mathcal{G}_{i j} \phi^{j^{\prime}}+V \mathcal{G}_{i j} \phi^{j^{\prime \prime}}-\frac{1}{2} V \partial_{i} \mathcal{G}_{k j} \phi^{k^{\prime}} \phi^{j^{\prime}} \\
-\frac{1}{2} e^{-2 \sum_{A} T_{A}} \partial_{i} G^{I J} q_{I} q_{J}-g^{2} \partial_{i} U=0 .
\end{array}
$$

\subsection{Magnetic ansatz}

In the magnetically charged case we take for the field strength

$$
F^{I}=p^{I} \theta^{1} \wedge \theta^{2}
$$

where the $p^{I}$ are magnetic charge densities. Note that $F^{I}$ is closed due to the Bianchi class A condition (A.4), so locally there exists a gauge potential $A^{I}$ such that $F^{I}=d A^{I}$. In the following we shall consider the case of solvegeometry, for which

$$
F^{I}=p^{I} d x \wedge d y, \quad A^{I}=p^{I} x d y .
$$

Using (A.7) the line element (3.1) becomes

$$
d s^{2}=-V(r) d t^{2}+\frac{d r^{2}}{V(r)}+e^{2\left(T_{1}(r)+z\right)} d x^{2}+e^{2\left(T_{2}(r)-z\right)} d y^{2}+e^{2 T_{3}(r)} d z^{2} .
$$

The Maxwell equations (2.6) are automatically satisfied by (3.12) and (3.13), while the nontrivial Einstein equations (2.5) read

$$
\begin{aligned}
\frac{V^{\prime \prime}}{2}+\frac{V^{\prime}}{2}\left(2 T_{1}^{\prime}+T_{3}^{\prime}\right) & =\frac{1}{3} e^{-4 T_{1}} G_{I J} p^{I} p^{J}-\frac{2}{3} g^{2} U, \\
2 T_{1}^{\prime \prime}+T_{3}^{\prime \prime}+2\left(T_{1}^{\prime}\right)^{2}+\left(T_{3}^{\prime}\right)^{2} & =-\mathcal{G}_{i j} \phi^{i^{\prime}} \phi^{j^{\prime}} \\
-V^{\prime} T_{1}^{\prime}-V\left(T_{1}^{\prime \prime}+T_{1}^{\prime}\left(2 T_{1}^{\prime}+T_{3}^{\prime}\right)\right) & =\frac{2}{3} e^{-4 T_{1}} G_{I J} p^{I} p^{J}+\frac{2}{3} g^{2} U, \\
-V^{\prime} T_{3}^{\prime}-V\left(T_{3}^{\prime \prime}+T_{3}^{\prime}\left(2 T_{1}^{\prime}+T_{3}^{\prime}\right)\right)-2 e^{-2 T_{3}} & =-\frac{1}{3} e^{-4 T_{1}} G_{I J} p^{I} p^{J}+\frac{2}{3} g^{2} U,
\end{aligned}
$$

where we have used the condition $T_{1}^{\prime}=T_{2}^{\prime}$ and the freedom to rescale $y$ in order to set $T_{1}=T_{2}$. The scalar field equations (2.7) become

$$
\begin{aligned}
V \mathcal{G}_{i j} \phi^{j^{\prime}} \sum_{A} T_{A}^{\prime}+V \frac{d \mathcal{G}_{i j}}{d r} \phi^{j^{\prime}}+V^{\prime} \mathcal{G}_{i j} \phi^{j^{\prime}} & +V \mathcal{G}_{i j} \phi^{j^{\prime \prime}}-\frac{1}{2} V \partial_{i} \mathcal{G}_{k j} \phi^{k^{\prime}} \phi^{j^{\prime}} \\
& -\frac{1}{2} \partial_{i} G_{I J} e^{-4 T_{1}} p^{I} p^{J}-g^{2} \partial_{i} U=0 .
\end{aligned}
$$




\section{Magnetic black hole in pure gauged supergravity}

In order to study the above equations in a simplified setting, we restrict our attention to pure gauged supergravity, i.e., the theory (2.1) without vector multiplets $(n=0)$. For a purely electric or magnetic configuration, the Chern-Simons term can be consistently truncated, and (2.1) boils down to

$$
e^{-1} \mathscr{L}=\frac{R}{2}-\frac{1}{4} F_{\mu \nu} F^{\mu \nu}-\Lambda,
$$

where $\Lambda=-6 g^{2}<0, F_{\mu \nu}=F_{\mu \nu}^{0}$ and we fixed $C_{000}$ in (2.3) and $V_{0}$ in (2.2) such that $G_{00}=1$ and $U=-6$.

The field strength (3.12) becomes simply $F_{x y}=p$, while the Einstein equations (3.14) reduce to

$$
\begin{aligned}
\frac{V^{\prime \prime}}{2}+\frac{V^{\prime}}{2}\left(2 T_{1}^{\prime}+T_{3}^{\prime}\right) & =\frac{1}{3} e^{-4 T_{1}} p^{2}-\frac{2}{3} \Lambda, \\
2 T_{1}^{\prime \prime}+T_{3}^{\prime \prime}+2\left(T_{1}^{\prime}\right)^{2}+\left(T_{3}^{\prime}\right)^{2} & =0 \\
-V^{\prime} T_{1}^{\prime}-V\left(T_{1}^{\prime \prime}+T_{1}^{\prime}\left(2 T_{1}^{\prime}+T_{3}^{\prime}\right)\right) & =\frac{2}{3} e^{-4 T_{1}} p^{2}+\frac{2}{3} \Lambda, \\
-V^{\prime} T_{3}^{\prime}-V\left(T_{3}^{\prime \prime}+T_{3}^{\prime}\left(2 T_{1}^{\prime}+T_{3}^{\prime}\right)\right)-2 e^{-2 T_{3}} & =-\frac{1}{3} e^{-4 T_{1}} p^{2}+\frac{2}{3} \Lambda .
\end{aligned}
$$

One easily checks that in the uncharged case $p=0$ the above equations are satisfied by the solvegeometry solution constructed in [14].

(4.2) can be easily solved by taking $T_{1}$ to be constant. ${ }^{4}$ With this assumption, a particular black hole solution is given by

$$
\begin{aligned}
d s^{2} & =-V(r) d t^{2}+\frac{d r^{2}}{V(r)}+\sqrt{\frac{p^{2}}{-\Lambda}}\left(e^{2 z} d x^{2}+e^{-2 z} d y^{2}\right)+\frac{r^{2}}{A} d z^{2} \\
F & =p d x \wedge d y
\end{aligned}
$$

with

$$
V(r)=-\frac{\Lambda}{2} r^{2}-2 A \ln \left(\frac{r}{B}\right),
$$

where $A$ and $B$ are two positive integration constants. It is worth noting that this solution is singular in the limit $p \rightarrow 0$, and it is thus disconnected from the one in [14]. The metric (4.3) and field strength (4.4) are invariant under the scale transformations

$$
t \rightarrow t / \nu, \quad r \rightarrow \nu r, \quad z \rightarrow z+\ln \alpha, \quad x \rightarrow \lambda x, \quad y \rightarrow \pm \lambda \alpha^{2} y,
$$

accompanied by

$$
p \rightarrow \pm \frac{p}{\lambda^{2} \alpha^{2}}, \quad A \rightarrow \nu^{2} A, \quad B \rightarrow \nu B .
$$

This can be used to set e.g. $p=B=1 / g$ without loss of generality. $B$ and the magnetic charge density $p$ are thus not true parameters of the solution, which is specified completely by choosing $A$. Notice that the scaling symmetries with $\nu=1, \lambda=1 / \alpha$ belong to the

\footnotetext{
${ }^{4}$ Note that this is not the case for the solution of [14].
} 
Lie group Sol. If the horizon is compactified (cf. [14] for details on the compactification procedure), the transformations in (4.6) involving $\alpha$ and $\lambda$ are broken down to a discrete subgroup ( $\alpha=\lambda^{-1}=e^{n a}$, where $a$ is the constant appearing in (II.22) of [14] and $n \in \mathbb{Z}$ ), which does no more allow to scale $p$ to any value. In this case, $p$ can become actually a genuine parameter of the black hole.

(4.3) exhibits anisotropic scaling. If the horizon is compactified, the geometry approaches asymptotically for $r \rightarrow \infty$ a torus bundle over $\mathrm{AdS}_{3}$. In $r=0$ there is a curvature singularity, since the Kretschmann scalar behaves as $R^{\mu \nu \rho \sigma} R_{\mu \nu \rho \sigma} \sim(\ln r) / r^{4}$ for $r \rightarrow 0$. Horizons are determined by the roots of the function $V(r)$, which diverges both for $r \rightarrow 0$ and $r \rightarrow+\infty$ and has a unique minimum in

$$
r=r_{\min }=\sqrt{\frac{2 A}{-\Lambda}}
$$

If $V\left(r_{\min }\right)>0$ the solution represents a naked singularity. For $V\left(r_{\min }\right)=0$, i.e., $A=3 e$, we have an extremal black hole, while for $V\left(r_{\min }\right)<0(A>3 e)$ there is an inner and an outer horizon and the solution is nonextremal.

Requiring the absence of conical singularities in the Euclidean section gives the Hawking temperature

$$
T=\frac{-\Lambda r_{\mathrm{h}}^{2}-2 A}{4 \pi r_{\mathrm{h}}}
$$

where $r_{\mathrm{h}}$ denotes the radial coordinate of the horizon. The entropy density can be computed by means of the Bekenstein-Hawking formula and is given by

$$
s=\frac{S}{V_{\text {solve }}}=\frac{\left(\ln \left(g r_{\mathrm{h}}\right)\right)^{1 / 2}}{12 g^{3}},
$$

where we set Newton's constant $G=1$, and $V_{\text {solve }}$ is the volume of the compactified manifold modelled on solvegeometry.

The standard Komar integral for the mass goes like $\Lambda r^{2}$ for large $r$ and thus diverges for $r \rightarrow+\infty$ due to the presence of the vacuum energy, as was to be expected. Moreover, there is no obvious background to subtract, and the conditions for the applicability of the Ashtekar-Magnon-Das formalism [26, 27] are not satisfied. In spite of these difficulties, we can associate a mass to the black hole (4.3) by simply integrating the first law. Since $p$ is not a dynamical parameter of the solution, we do not expect a term containing the variation of the magnetic charge in the first law. The mass density $m$ satisfies thus

$$
d m=T d s,
$$

which gives (up to an integration constant, that can be fixed by requiring e.g. the extremal solution to have zero energy)

$$
m=\frac{r_{\mathrm{h}}}{16 \pi g\left(\ln \left(g r_{\mathrm{h}}\right)\right)^{1 / 2}}=\frac{1}{8 \pi g^{2}} \sqrt{\frac{A}{6}} .
$$

Notice that in five dimensions, magnetic charge is actually carried by strings rather than by point particles, since the string world volume naturally couples to an electric two-form 
potential, which is dual to the magnetic ansatz (3.12). The latter is characteristic of a charged string along the $z$-direction. In this sense, the solution constructed in this section is perhaps more correctly referred to as a black string rather than a black hole. In spite of this, we shall use the terminology 'black hole', since the near-horizon geometry of the extremal limit of (4.3) contains an $\mathrm{AdS}_{2}$ factor (it is $\mathrm{AdS}_{2} \times \mathrm{Sol}$ ) instead of $\mathrm{AdS}_{3},{ }^{5}$ which would be typical for extremal black strings. Note also that, if we think of (4.3) as a black string, then this is not straightforwardly related to a black hole in four dimensions, since the explicit $z$-dependence of the metric prevents a canonical Kaluza-Klein reduction along $z$.

To close this section, we remark that a generalization of the solution (4.3), (4.4) as well as the one of [14] to the stu model of $N=2$ gauged supergravity, together with a numerical analysis of the equations of motion (4.2), is currently under investigation.

\section{Existence of static, Sol-invariant BPS solutions}

A simpler method to construct solutions to a given supergravity theory is based on solving the Killing spinor equations. These are of first order, and are generically much easier to solve than the full second order equations of motion. At least in the case where the Killing vector constructed as a bilinear from the Killing spinor is timelike, the latter are implied by the Killing spinor equations [28].

The supersymmetry variations for the gravitino $\psi_{\mu}$ and the gauginos $\lambda_{i}$ in a bosonic background are given by (see e.g. [29])

$$
\begin{aligned}
\delta \psi_{\mu} & =\left[\mathcal{D}_{\mu}+\frac{i}{8} h_{I}\left(\Gamma_{\mu}^{\nu \rho}-4 \delta_{\mu}{ }^{\nu} \Gamma^{\rho}\right) F_{\nu \rho}^{I}+\frac{g}{2} \Gamma_{\mu} h^{I} V_{I}-\frac{3 i}{2} g V_{I} A_{\mu}^{I}\right] \epsilon, \\
\delta \lambda_{i} & =\left[\frac{3}{8} \Gamma^{\mu \nu} F_{\mu \nu}^{I} \partial_{i} h_{I}-\frac{i}{2} \mathcal{G}_{i j} \Gamma^{\mu} \partial_{\mu} \phi^{j}+\frac{3 i}{2} g V_{I} \partial_{i} h^{I}\right] \epsilon
\end{aligned}
$$

where $\epsilon$ is the supersymmetry parameter, $h_{I}=\frac{1}{6} C_{I J K} h^{J} h^{K}$ and $\mathcal{D}_{\mu}$ denotes the Lorentzcovariant derivative. ${ }^{6}$ The vanishing of the gravitino supersymmetry transformations (5.1) leads to the Killing spinor equations, whose integrability conditions imply a set of constraints for the metric and the matter fields. Given $\delta \psi_{\mu} \equiv \hat{\mathcal{D}}_{\mu} \epsilon=0$, the first integrability conditions read

$$
\hat{\mathcal{R}}_{\mu \nu} \epsilon \equiv\left[\hat{\mathcal{D}}_{\mu}, \hat{\mathcal{D}}_{\nu}\right] \epsilon=0
$$

which is a set of algebraic equations that admit a nontrivial solution $\epsilon$ iff $\operatorname{det}\left(\hat{\mathcal{R}}_{\mu \nu}\right)=0$.

In what follows we shall specify to solvegeometry with electric or magnetic ansatz. For the metric (3.13) the tetrad can be chosen as

$$
e_{t}^{0}=\sqrt{V}, \quad e_{x}^{1}=e^{T_{1}+z}, \quad e_{y}^{2}=e^{T_{2}-z}, \quad e_{z}^{3}=e^{T_{3}}, \quad e_{r}^{4}=\frac{1}{\sqrt{V}} .
$$

\footnotetext{
${ }^{5}$ As we said, $\mathrm{AdS}_{3}$ occurs for $r \rightarrow \infty$.

${ }^{6}$ Our conventions are $\mathcal{D}_{\mu}=\partial_{\mu}+\frac{1}{4} \omega_{\mu}^{a b} \Gamma_{a b},\left\{\Gamma^{a}, \Gamma^{b}\right\}=2 \eta^{a b}, \Gamma^{a_{1} a_{2} \ldots a_{n}}=\Gamma^{\left[a_{1}\right.} \Gamma^{a_{2}} \ldots \Gamma^{\left.a_{n}\right]}$, where we antisymmetrize with unit weight.
} 


\subsection{Electric ansatz}

In the case of solvegeometry and electric ansatz, the vanishing of the gravitino variations (5.1) leads to

$$
\begin{aligned}
& {\left[\partial_{t}+\frac{V^{\prime}}{4} \Gamma_{04}+\frac{i}{2} h_{I} F_{r t}^{I} \sqrt{V} \Gamma_{4}+\frac{g}{2} V_{I} h^{I} \sqrt{V} \Gamma_{0}-i \frac{3 g}{2} V_{I} A_{t}^{I}\right] \epsilon=0,} \\
& {\left[\partial_{r}+\frac{i}{2} h_{I} F_{r t}^{I} \frac{1}{\sqrt{V}} \Gamma_{0}+\frac{g}{2} V_{I} h^{I} \frac{1}{\sqrt{V}} \Gamma_{4}\right] \epsilon=0,} \\
& {\left[\partial_{x}+e^{T_{1}+z}\left(\frac{1}{2} \sqrt{V} T_{1}^{\prime} \Gamma_{14}+\frac{1}{2} e^{-T_{3}} \Gamma_{13}-\frac{i}{4} h_{I} F_{r t}^{I} \Gamma_{014}+\frac{g}{2} V_{I} h^{I} \Gamma_{1}\right)\right] \epsilon=0,} \\
& {\left[\partial_{y}+e^{T_{2}-z}\left(\frac{1}{2} \sqrt{V} T_{2}^{\prime} \Gamma_{24}-\frac{1}{2} e^{-T_{3}} \Gamma_{23}-\frac{i}{4} h_{I} F_{r t}^{I} \Gamma_{024}+\frac{g}{2} V_{I} h^{I} \Gamma_{2}\right)\right] \epsilon=0,} \\
& {\left[\partial_{z}+e^{T_{3}}\left(\frac{1}{2} \sqrt{V} T_{3}^{\prime} \Gamma_{34}-\frac{i}{4} h_{I} F_{r t}^{I} \Gamma_{034}+\frac{g}{2} V_{I} h^{I} \Gamma_{3}\right)\right] \epsilon=0 .}
\end{aligned}
$$

The integrability conditions (5.3) with $(\mu, \nu)$ equal to $(t, x),(t, y)$ and $(t, z)$ are, respectively,

$$
\begin{aligned}
& {\left[\frac{1}{2} V^{\prime} T_{1}^{\prime}-g^{2}\left(V_{I} h^{I}\right)^{2}+i \sqrt{V} T_{1}^{\prime} h_{I} F_{r t}^{I} \Gamma_{0}+i g V_{I} h^{I} h_{J} F_{r t}^{J} \Gamma_{04}\right] \epsilon=0,} \\
& {\left[\frac{1}{2} V^{\prime} T_{2}^{\prime}-g^{2}\left(V_{I} h^{I}\right)^{2}+i \sqrt{V} T_{2}^{\prime} h_{I} F_{r t}^{I} \Gamma_{0}+i g V_{I} h^{I} h_{J} F_{r t}^{J} \Gamma_{04}\right] \epsilon=0,} \\
& {\left[\frac{1}{2} V^{\prime} T_{3}^{\prime}-g^{2}\left(V_{I} h^{I}\right)^{2}+i \sqrt{V} T_{3}^{\prime} h_{I} F_{r t}^{I} \Gamma_{0}+i g V_{I} h^{I} h_{J} F_{r t}^{J} \Gamma_{04}\right] \epsilon=0,}
\end{aligned}
$$

while for $(x, y),(x, z)$ and $(y, z)$ we have

$$
\begin{gathered}
{\left[V T_{1}^{\prime} T_{2}^{\prime}-g^{2}\left(V_{I} h^{I}\right)^{2}+\frac{1}{4}\left(h_{I} F_{r t}^{I}\right)^{2}-e^{-2 T_{3}}-\frac{i}{2} \sqrt{V}\left(T_{1}^{\prime}+T_{2}^{\prime}\right) h_{I} F_{r t}^{I} \Gamma_{0}\right.} \\
\left.-i g V_{I} h^{I} h_{J} F_{r t}^{J} \Gamma_{04}\right] \epsilon=0, \\
{\left[V T_{1}^{\prime} T_{3}^{\prime}-g^{2}\left(V_{I} h^{I}\right)^{2}+\frac{1}{4}\left(h_{I} F_{r t}^{I}\right)^{2}+e^{-2 T_{3}}-\frac{i}{2} \sqrt{V}\left(T_{1}^{\prime}+T_{3}^{\prime}\right) h_{I} F_{r t}^{I} \Gamma_{0}\right.} \\
\left.-i g V_{I} h^{I} h_{J} F_{r t}^{J} \Gamma_{04}+\sqrt{V}\left(T_{1}^{\prime}-T_{3}^{\prime}\right) e^{-T_{3}} \Gamma_{34}\right] \epsilon=0, \\
{\left[V T_{2}^{\prime} T_{3}^{\prime}-g^{2}\left(V_{I} h^{I}\right)^{2}+\frac{1}{4}\left(h_{I} F_{r t}^{I}\right)^{2}+e^{-2 T_{3}}-\frac{i}{2} \sqrt{V}\left(T_{2}^{\prime}+T_{3}^{\prime}\right) h_{I} F_{r t}^{I} \Gamma_{0}\right.} \\
\left.-i g V_{I} h^{I} h_{J} F_{r t}^{J} \Gamma_{04}-\sqrt{V}\left(T_{2}^{\prime}-T_{3}^{\prime}\right) e^{-T_{3}} \Gamma_{34}\right] \epsilon=0 .
\end{gathered}
$$

The difference of eqs. (5.6) taken in (all the three possible) pairs leads to

$$
\begin{aligned}
& \left(T_{1}^{\prime}-T_{2}^{\prime}\right)\left[\frac{1}{2} V^{\prime}+i \sqrt{V} h_{I} F_{r t}^{I} \Gamma_{0}\right] \epsilon=0, \\
& \left(T_{1}^{\prime}-T_{3}^{\prime}\right)\left[\frac{1}{2} V^{\prime}+i \sqrt{V} h_{I} F_{r t}^{I} \Gamma_{0}\right] \epsilon=0, \\
& \left(T_{2}^{\prime}-T_{3}^{\prime}\right)\left[\frac{1}{2} V^{\prime}+i \sqrt{V} h_{I} F_{r t}^{I} \Gamma_{0}\right] \epsilon=0,
\end{aligned}
$$


whereas $(x, y)-(x, z)$ and $(x, y)-(y, z)$ read

$$
\begin{aligned}
& {\left[V T_{1}^{\prime}\left(T_{2}^{\prime}-T_{3}^{\prime}\right)-2 e^{-2 T_{3}}-\frac{i}{2} \sqrt{V}\left(T_{2}^{\prime}-T_{3}^{\prime}\right) h_{I} F_{r t}^{I} \Gamma_{0}-\sqrt{V}\left(T_{1}^{\prime}-T_{3}^{\prime}\right) e^{-T_{3}} \Gamma_{34}\right] \epsilon=0,} \\
& {\left[V T_{2}^{\prime}\left(T_{1}^{\prime}-T_{3}^{\prime}\right)-2 e^{-2 T_{3}}-\frac{i}{2} \sqrt{V}\left(T_{1}^{\prime}-T_{3}^{\prime}\right) h_{I} F_{r t}^{I} \Gamma_{0}+\sqrt{V}\left(T_{2}^{\prime}-T_{3}^{\prime}\right) e^{-T_{3}} \Gamma_{34}\right] \epsilon=0 .}
\end{aligned}
$$

We can distinguish between two different cases in which (5.8) hold.

- Case A

$$
T_{1}^{\prime}=T_{2}^{\prime}=T_{3}^{\prime} .
$$

In this case (5.9) leads directly to the trivial solution $\epsilon=0$.

- Case B

$$
\left[\frac{1}{2} V^{\prime}+i \sqrt{V} h_{I} F_{r t}^{I} \Gamma_{0}\right] \epsilon=0 .
$$

Writing this condition schematically as $\mathcal{M} \epsilon=0$, a necessary condition to have nontrivial solutions is $\operatorname{det} \mathcal{M}=0$, and thus

$$
\frac{1}{2} V^{\prime}= \pm \sqrt{V} h_{I} F_{r t}^{I},
$$

which, once plugged back into (5.11) gives the projection

$$
\Gamma_{0} \epsilon= \pm i \epsilon
$$

Using (5.13) in (5.9), we get

$$
\begin{aligned}
& {\left[V T_{1}^{\prime}\left(T_{2}^{\prime}-T_{3}^{\prime}\right)-2 e^{-2 T_{3}} \pm \frac{1}{2} \sqrt{V}\left(T_{2}^{\prime}-T_{3}^{\prime}\right) h_{I} F_{r t}^{I}-\sqrt{V}\left(T_{1}^{\prime}-T_{3}^{\prime}\right) e^{-T_{3}} \Gamma_{34}\right] \epsilon=0} \\
& {\left[V T_{2}^{\prime}\left(T_{1}^{\prime}-T_{3}^{\prime}\right)-2 e^{-2 T_{3}} \pm \frac{1}{2} \sqrt{V}\left(T_{1}^{\prime}-T_{3}^{\prime}\right) h_{I} F_{r t}^{I}+\sqrt{V}\left(T_{2}^{\prime}-T_{3}^{\prime}\right) e^{-T_{3}} \Gamma_{34}\right] \epsilon=0}
\end{aligned}
$$

To have nontrivial solutions, the determinants of the two coefficient matrices in these linear systems must vanish, leading to $T_{1}^{\prime}=T_{3}^{\prime}$ and $T_{2}^{\prime}=T_{3}^{\prime}$, which brings us back to case A.

We can thus state the following

Proposition 1. There are no static, Sol-invariant solutions to the Killing spinor equations with solvegeometry spatial cross-sections at fixed $r$ and purely electric field strengths. 


\subsection{Magnetic ansatz}

In this case, the Killing spinor equations become

$$
\begin{aligned}
& {\left[\partial_{t}+\frac{V^{\prime}}{4} \Gamma_{04}+\frac{i}{4} h_{I} p^{I} \sqrt{V} e^{-T_{1}-T_{2}} \Gamma_{012}+\frac{g}{2} V_{I} h^{I} \sqrt{V} \Gamma_{0}\right] \epsilon=0,} \\
& {\left[\partial_{r}+\frac{i}{4} h_{I} p^{I} \frac{1}{\sqrt{V}} e^{-T_{1}-T_{2}} \Gamma_{124}+\frac{g}{2} V_{I} h^{I} \frac{1}{\sqrt{V}} \Gamma_{4}\right] \epsilon=0,} \\
& {\left[\partial_{x}+e^{T_{1}+z}\left(\frac{1}{2} \sqrt{V} T_{1}^{\prime} \Gamma_{14}+\frac{1}{2} e^{-T_{3}} \Gamma_{13}-\frac{i}{2} h_{I} p^{I} e^{-T_{1}-T_{2}} \Gamma_{2}+\frac{g}{2} V_{I} h^{I} \Gamma_{1}\right)\right] \epsilon=0,} \\
& {\left[\partial_{y}+e^{T_{2}-z}\left(\frac{1}{2} \sqrt{V} T_{2}^{\prime} \Gamma_{24}-\frac{1}{2} e^{-T_{3}} \Gamma_{23}+\frac{i}{2} h_{I} p^{I} e^{-T_{1}-T_{2}} \Gamma_{1}+\frac{g}{2} V_{I} h^{I} \Gamma_{2}\right)-i \frac{3 g}{2} V_{I} p^{I} x\right] \epsilon=0,} \\
& {\left[\partial_{z}+e^{T_{3}}\left(\frac{1}{2} \sqrt{V} T_{3}^{\prime} \Gamma_{34}+\frac{i}{4} h_{I} p^{I} e^{-T_{1}-T_{2}} \Gamma_{123}+\frac{g}{2} V_{I} h^{I} \Gamma_{3}\right)\right] \epsilon=0 .}
\end{aligned}
$$

We have thus the following first integrability conditions:

- $(\mathrm{t}, \mathrm{x})$

$$
\begin{aligned}
{\left[\frac{1}{2} V^{\prime} T_{1}^{\prime}-g^{2}\left(V_{I} h^{I}\right)^{2}+\frac{i}{2} h_{I} p^{I} \sqrt{V} T_{1}^{\prime} e^{-T_{1}-T_{2}} \Gamma_{124}\right.} & +\frac{i}{2} h_{I} p^{I} e^{-T_{1}-T_{2}-T_{3}} \Gamma_{123} \\
& \left.+i g h_{I} p^{I} V_{J} h^{J} e^{-T_{1}-T_{2}} \Gamma_{12}\right] \epsilon=0,
\end{aligned}
$$

- $(\mathrm{t}, \mathrm{y})$

$$
\begin{aligned}
{\left[\frac{1}{2} V^{\prime} T_{2}^{\prime}-g^{2}\left(V_{I} h^{I}\right)^{2}+\frac{i}{2} h_{I} p^{I} \sqrt{V} T_{2}^{\prime} e^{-T_{1}-T_{2}} \Gamma_{124}\right.} & -\frac{i}{2} h_{I} p^{I} e^{-T_{1}-T_{2}-T_{3}} \Gamma_{123} \\
& \left.+i g h_{I} p^{I} V_{J} h^{J} e^{-T_{1}-T_{2}} \Gamma_{12}\right] \epsilon=0
\end{aligned}
$$

- $(\mathrm{t}, \mathrm{z})$

$$
\left[\frac{1}{2} V^{\prime} T_{3}^{\prime}-g^{2}\left(V_{I} h^{I}\right)^{2}-i g h_{I} p^{I} V_{J} h^{J} e^{-T_{1}-T_{2}} \Gamma_{12}-\frac{1}{4}\left(h_{I} p^{I}\right)^{2} e^{-2\left(T_{1}+T_{2}\right)}\right] \epsilon=0,
$$

- $(\mathrm{x}, \mathrm{y})$

$$
\begin{aligned}
& {\left[V T_{1}^{\prime} T_{2}^{\prime}-g^{2}\left(V_{I} h^{I}\right)^{2}+\left(h_{I} p^{I}\right)^{2} e^{-2\left(T_{1}+T_{2}\right)}-e^{-2 T_{3}}\right.} \\
& \left.\quad-i h_{I} p^{I} \sqrt{V}\left(T_{1}^{\prime}+T_{2}^{\prime}\right) e^{-T_{1}-T_{2}} \Gamma_{124}-3 i g V_{I} p^{I} e^{-T_{1}-T_{2}} \Gamma_{12}\right] \epsilon=0,
\end{aligned}
$$

- $(\mathrm{x}, \mathrm{z})$

$$
\begin{aligned}
{\left[V T_{1}^{\prime} T_{3}^{\prime}-g^{2}\left(V_{I} h^{I}\right)^{2}+\right.} & e^{-2 T_{3}}+\sqrt{V}\left(T_{1}^{\prime}-T_{3}^{\prime}\right) e^{-T_{3}} \Gamma_{34}-i h_{I} p^{I} e^{-T_{1}-T_{2}-T_{3}} \Gamma_{123} \\
& \left.+\frac{i}{2} h_{I} p^{I} \sqrt{V} T_{1}^{\prime} e^{-T_{1}-T_{2}} \Gamma_{124}+i g h_{I} p^{I} V_{J} h^{J} e^{-T_{1}-T_{2}} \Gamma_{12}\right] \epsilon=0,
\end{aligned}
$$


- $(\mathrm{y}, \mathrm{z})$

$$
\begin{aligned}
{\left[V T_{2}^{\prime} T_{3}^{\prime}-g^{2}\left(V_{I} h^{I}\right)^{2}+\right.} & e^{-2 T_{3}}-\sqrt{V}\left(T_{2}^{\prime}-T_{3}^{\prime}\right) e^{-T_{3}} \Gamma_{34}+i h_{I} p^{I} e^{-T_{1}-T_{2}-T_{3}} \Gamma_{123} \\
& \left.+\frac{i}{2} h_{I} p^{I} \sqrt{V} T_{2}^{\prime} e^{-T_{1}-T_{2}} \Gamma_{124}+i g h_{I} p^{I} V_{J} h^{J} e^{-T_{1}-T_{2}} \Gamma_{12}\right] \epsilon=0,
\end{aligned}
$$

- $(\mathrm{r}, \mathrm{t})$

$$
\begin{array}{r}
{\left[\frac{1}{2} V^{\prime \prime}-g^{2}\left(V_{I} h^{I}\right)^{2}-\frac{1}{4}\left(h_{I} p^{I}\right)^{2} e^{-2\left(T_{1}+T_{2}\right)}-\frac{i}{2} h_{I} p^{I} \sqrt{V}\left(T_{1}^{\prime}+T_{2}^{\prime}\right) e^{-T_{1}-T_{2}} \Gamma_{124}\right.} \\
-\frac{i}{2} \partial_{r}\left(h_{I} p^{I}\right) \sqrt{V} e^{-T_{1}-T_{2}} \Gamma_{124}+g \partial_{r}\left(V_{I} h^{I}\right) \sqrt{V} \Gamma_{4} \\
\left.-i g h_{I} p^{I} V_{J} h^{J} e^{-T_{1}-T_{2}} \Gamma_{12}\right] \epsilon=0,
\end{array}
$$

- $(\mathrm{r}, \mathrm{x})$

$$
\begin{aligned}
{\left[V T_{1}^{\prime \prime}+V T_{1}^{\prime 2}-i \partial_{r}\left(h_{I} p^{I}\right)\right.} & \sqrt{V} e^{-T_{1}-T_{2}} \Gamma_{124}+g \partial_{r}\left(V_{I} h^{I}\right) \sqrt{V} \Gamma_{4} \\
& \left.-\frac{i}{2} h_{I} p^{I} \sqrt{V}\left(T_{1}^{\prime}-2 T_{2}^{\prime}\right) e^{-T_{1}-T_{2}} \Gamma_{124}\right] \epsilon=0,
\end{aligned}
$$

- $(\mathrm{r}, \mathrm{y})$

$$
\begin{aligned}
{\left[V T_{2}^{\prime \prime}+V{T_{2}^{\prime}}^{2}-i \partial_{r}\left(h_{I} p^{I}\right)\right.} & \sqrt{V} e^{-T_{1}-T_{2}} \Gamma_{124}+g \partial_{r}\left(V_{I} h^{I}\right) \sqrt{V} \Gamma_{4} \\
& \left.-\frac{i}{2} h_{I} p^{I} \sqrt{V}\left(T_{2}^{\prime}-2 T_{1}^{\prime}\right) e^{-T_{1}-T_{2}} \Gamma_{124}\right] \epsilon=0,
\end{aligned}
$$

- $(\mathrm{r}, \mathrm{z})$

$$
\begin{aligned}
{\left[V T_{3}^{\prime \prime}+V T_{3}^{\prime 2}+\frac{i}{2} \partial_{r}\left(h_{I} p^{I}\right)\right.} & \sqrt{V} e^{-T_{1}-T_{2}} \Gamma_{124}+g \partial_{r}\left(V_{I} h^{I}\right) \sqrt{V} \Gamma_{4} \\
& \left.-\frac{i}{2} h_{I} p^{I} \sqrt{V}\left(T_{1}^{\prime}+T_{2}^{\prime}\right) e^{-T_{1}-T_{2}} \Gamma_{124}\right] \epsilon=0 .
\end{aligned}
$$

From the vanishing of the gaugino variation (5.2) one gets

$$
\left[\frac{1}{3} \mathcal{G}_{i j} \sqrt{V} \partial_{r} \phi^{j} \Gamma_{4}-g \partial_{i}\left(V_{I} h^{I}\right)+\frac{i}{2} \partial_{i}\left(h_{I} p^{I}\right) e^{-T_{1}-T_{2}} \Gamma_{12}\right] \epsilon=0 .
$$

The combination $(5.19)+(5.20)-(5.15)-(5.16)$ gives

$$
\left[\left(T_{1}^{\prime}+T_{2}^{\prime}\right)\left(V T_{3}^{\prime}-\frac{1}{2} V^{\prime}\right)+2 e^{-2 T_{3}}+\sqrt{V}\left(T_{1}^{\prime}-T_{2}^{\prime}\right) e^{-T_{3}} \Gamma_{34}\right] \epsilon=0 .
$$

The determinant of the coefficient matrix of this linear system vanishes if

$$
\left(T_{1}^{\prime}+T_{2}^{\prime}\right)\left(V T_{3}^{\prime}-\frac{1}{2} V^{\prime}\right)+2 e^{-2 T_{3}}=0 \quad \wedge \quad \sqrt{V}\left(T_{1}^{\prime}-T_{2}^{\prime}\right) e^{-T_{3}}=0,
$$


which implies

$$
T_{1}^{\prime}\left(V T_{3}^{\prime}-\frac{1}{2} V^{\prime}\right)+e^{-2 T_{3}}=0, \quad T_{1}^{\prime}=T_{2}^{\prime} .
$$

From the combination $(5.22)-(5.23)+(5.19)-(5.20)+2 \cdot((5.15)-(5.16))$ we obtain

$$
\left[V\left(T_{1}^{\prime \prime}-T_{2}^{\prime \prime}\right)+\left(T_{1}^{\prime}-T_{2}^{\prime}\right)\left(V\left(T_{1}^{\prime}+T_{2}^{\prime}+T_{3}^{\prime}\right)+V^{\prime}\right)+\sqrt{V}\left(T_{1}^{\prime}+T_{2}^{\prime}-2 T_{3}^{\prime}\right) e^{-T_{3}} \Gamma_{34}\right] \epsilon=0
$$

Using $T_{1}^{\prime}=T_{2}^{\prime}$, it turns out that the vanishing of the determinant associated to (5.29) requires $T_{3}^{\prime}=T_{1}^{\prime}$. (5.15) $-(5.16)$ yields

$$
i h_{I} p^{I} e^{-T_{1}-T_{2}-T_{3}} \Gamma_{123} \epsilon=0,
$$

and thus

$$
h_{I} p^{I}=0 .
$$

Taking into account the above results and defining $T^{\prime} \equiv T_{1}^{\prime}=T_{2}^{\prime}=T_{3}^{\prime}$, the first integrability conditions become

- $(\mathrm{t}, \mathrm{x}),(\mathrm{t}, \mathrm{y}),(\mathrm{t}, \mathrm{z})$

$$
\left[\frac{1}{2} V^{\prime} T^{\prime}-g^{2}\left(V_{I} h^{I}\right)^{2}\right] \epsilon=0,
$$

- $(\mathrm{x}, \mathrm{y})$

$$
\left[V T^{\prime 2}-g^{2}\left(V_{I} h^{I}\right)^{2}-e^{-2 T_{3}}-3 i g V_{I} p^{I} e^{-T_{1}-T_{2}} \Gamma_{12}\right] \epsilon=0
$$

- $(\mathrm{x}, \mathrm{z}),(\mathrm{y}, \mathrm{z})$

$$
\left[V T^{\prime 2}-g^{2}\left(V_{I} h^{I}\right)^{2}+e^{-2 T_{3}}\right] \epsilon=0
$$

- $(\mathrm{r}, \mathrm{t})$

$$
\left[\frac{1}{2} V^{\prime \prime}-g^{2}\left(V_{I} h^{I}\right)^{2}+g \partial_{r}\left(V_{I} h^{I}\right) \sqrt{V} \Gamma_{4}\right] \epsilon=0
$$

- $(\mathrm{r}, \mathrm{x}),(\mathrm{r}, \mathrm{y}),(\mathrm{r}, \mathrm{z})$

$$
\left[V T^{\prime \prime}+V T^{\prime 2}+g \partial_{r}\left(V_{I} h^{I}\right) \sqrt{V} \Gamma_{4}\right] \epsilon=0 .
$$

$(5.33)-(5.34)$ leads to

$$
\left[2 e^{-2 T_{3}}+3 i g V_{I} p^{I} e^{-T_{1}-T_{2}} \Gamma_{12}\right] \epsilon=0
$$

which implies the Dirac-type quantization condition

$$
V_{I} p^{I}=\sigma_{1} \frac{2}{3 g} e^{T_{1}+T_{2}-2 T_{3}},
$$


where $\sigma_{1}= \pm 1$. Plugging this back into (5.37) gives

$$
\Gamma_{12} \epsilon=i \sigma_{1} \epsilon
$$

With (5.39), the gaugino equation (5.25) becomes

$$
\left[\frac{1}{3} \mathcal{G}_{i j} \sqrt{V} \partial_{r} \phi^{j} \Gamma_{4}-g \partial_{i}\left(V_{I} h^{I}\right)-\sigma_{1} \frac{1}{2} \partial_{i}\left(h_{I} p^{I}\right) e^{-T_{1}-T_{2}}\right] \epsilon=0
$$

If the scalar fields were constant, $\partial_{r} \phi^{j}=0 \forall j$, this would imply

$$
g \partial_{i}\left(V_{I} h^{I}\right)+\sigma_{1} \frac{1}{2} \partial_{i}\left(h_{I} p^{I}\right) e^{-T_{1}-T_{2}}=0,
$$

and thus $T_{1}$ and $T_{2}$ must be constant as well, which leads to a contradiction with the first equation of (5.27). Note that this conclusion is valid provided $\partial_{i}\left(V_{I} h^{I}\right)$ and $\partial_{i}\left(h_{I} p^{I}\right)$ do not both vanish. In the latter case, however, using one of the very special geometry relations, we have

$$
0=\mathcal{G}^{i j} \partial_{i}\left(h_{I} p^{I}\right) \partial_{j}\left(h_{J} p^{J}\right)=\frac{4}{9} G_{I J} p^{I} p^{J}-\frac{2}{3} h_{I} p^{I} h_{J} p^{J}=\frac{4}{9} G_{I J} p^{I} p^{J},
$$

where the last step follows from (5.31). Since $G_{I J}$ is positive definite, (5.42) leads to a contradiction. If $\partial_{r} \phi^{i} \neq 0$ for at least one $i$, one can multiply (5.40) with $\partial_{r} \phi^{i}$ and sum over $i$ to get $^{7}$

$$
\left[\frac{1}{3} \mathcal{G}_{i j} \sqrt{V} \partial_{r} \phi^{i} \partial_{r} \phi^{j} \Gamma_{4}-g \partial_{r}\left(V_{I} h^{I}\right)\right] \epsilon=0 .
$$

We see immediately that one needs $\partial_{r}\left(V_{I} h^{I}\right) \neq 0$, since otherwise $\mathcal{G}_{i j} \partial_{r} \phi^{i} \partial_{r} \phi^{j}=0$, which is impossible because $\mathcal{G}_{i j}$ is a definite matrix.

To proceed, we require the determinants associated to the linear systems (5.35) and (5.36) to vanish, which implies the projection condition $\Gamma_{4} \epsilon=-\sigma_{2} \epsilon\left(\sigma_{2}= \pm 1\right)$ as well as

$$
\begin{aligned}
& \sigma_{2} g \partial_{r}\left(V_{I} h^{I}\right) \sqrt{V}=\frac{1}{2} V^{\prime \prime}-g^{2}\left(V_{I} h^{I}\right)^{2}, \\
& \sigma_{2} g \partial_{r}\left(V_{I} h^{I}\right) \sqrt{V}=V T^{\prime \prime}+V T^{\prime 2} .
\end{aligned}
$$

Deriving the prefactor of $\epsilon$ in (5.32) w.r.t. $r$, one obtains, using also (5.44) and (5.32),

$$
\begin{aligned}
& 2 g^{2}\left(V_{J} h^{J}\right) \partial_{r}\left(V_{I} h^{I}\right)=\frac{1}{2}\left(V^{\prime \prime} T^{\prime}+V^{\prime} T^{\prime \prime}\right) \\
& \quad=\left(\sigma_{2} g \partial_{r}\left(V_{I} h^{I}\right) \sqrt{V}+g^{2}\left(V_{I} h^{I}\right)^{2}\right) T^{\prime}+\frac{1}{2} V^{\prime}\left(\sigma_{2} g \partial_{r}\left(V_{I} h^{I}\right) \frac{1}{\sqrt{V}}-T^{\prime 2}\right) \\
& \quad=\sigma_{2} g \partial_{r}\left(V_{I} h^{I}\right)\left(\sqrt{V} T^{\prime}+\frac{V^{\prime}}{2 \sqrt{V}}\right)
\end{aligned}
$$

Thus, since $\partial_{r}\left(V_{I} h^{I}\right) \neq 0$,

$$
g\left(V_{I} h^{I}\right)=\sigma_{2} \frac{1}{2}\left(\sqrt{V} T^{\prime}+\frac{V^{\prime}}{2 \sqrt{V}}\right)
$$

\footnotetext{
${ }^{7}$ Notice that $\partial_{r}\left(h_{I} p^{I}\right)=0$.
} 
Derive this w.r.t. $r$ and then subtract the sum of the two eqs. in (5.44), divided by two, to get

$$
0=\sigma_{2} \frac{1}{2}\left(\frac{V^{\prime} T^{\prime}}{\sqrt{V}}-\frac{V^{\prime 2}}{4 V^{3 / 2}}-\sqrt{V}{T^{\prime}}^{2}\right)=-\sigma_{2} \frac{\sqrt{V}}{2}\left(\frac{V^{\prime}}{2 V}-T^{\prime}\right)^{2}=-\sigma_{2} \frac{1}{2 V^{3 / 2} T^{\prime 2}} e^{-4 T_{3}}
$$

where the last step follows from the first eq. of (5.27). Evidently, (5.47) leads to a contradiction, which implies

Proposition 2. There are no static, Sol-invariant solutions to the Killing spinor equations with solvegeometry spatial cross-sections at fixed $r$ and purely magnetic field strengths.

In particular, there is no BPS limit of the black hole constructed in section 4. Note in this context that rotating supersymmetric Nil and $\widetilde{\mathrm{SL}}(2, \mathbb{R})$ near-horizon geometries were found in [30]. Moreover, it is worth mentioning that the near-horizon limit of all supersymmetric extremal black holes in gauged (and ungauged) five-dimensional supergravity coupled to abelian vector multiplets must admit an $\mathrm{SL}(2, \mathbb{R})$ symmetry group [31]. This follows from an index theory argument and extends earlier results of [32] for minimal gauged supergravity.

\section{Attractor mechanism}

According to the attractor mechanism [17-21], the entropy of an extremal black hole and the scalar fields on the event horizon are insensitive to the asymptotic values of the moduli and depend only on the electric and magnetic charges. ${ }^{8}$ This phenomenon was first discovered in four-dimensional ungauged supergravity for BPS black holes [17] and proved in [21]. It was subsequently extended to higher dimensions, non-supersymmetric or rotating solutions, and gauged supergravities, cf. [33-41] for an (incomplete) list of references. In particular, the generalization of the proof of [21] to five dimensions was given in [42], and to general spatial and worldvolume dimensions in [43]. Notice that [42, 43] are valid for asymptotically flat black holes only. There is no generalization that covers at the same time dimensions higher than four and non-flat asymptotics.

A recurrent feature in all these cases is that the scalar configuration on the horizon can be determined by extremizing an effective potential and that the entropy is given by the value of this potential at its extremum.

In this section, we study the attractor mechanism for extremal static black holes with nil- or solvegeometry horizons in the theory (2.1). It will turn out that there are no such attractors for purely electric field strengths, while in the magnetic case there are attractor geometries, for which we explicitely determine the effective potential $V_{\text {eff, }}$ which contains the charges as well as the scalar potential of the gauged supergravity theory.

\footnotetext{
${ }^{8}$ This is valid in the absence of flat directions in the effective potential for the scalars. In the generic situation not all the moduli are stabilized on the horizon. Nevertheless, the black hole entropy is still independent of the values of the scalars that are not stabilized.
} 


\subsection{Magnetic ansatz}

As a first step to extend the black hole solution (4.3) to the matter-coupled case, we consider the near-horizon limit of the ansatz (3.1). Following closely the argument presented in [41], we are interested in magnetically charged, static and extremal black holes with Sol horizon, but without referring to any particular model of very special geometry. Extremality implies that the near-horizon geometry is the product manifold $\mathrm{AdS}_{2} \times$ Sol. Assuming the horizon to be located at $r=0$, we have thus for $r \rightarrow 0$

$$
V(r) \sim\left(\frac{r}{r_{\mathrm{AdS}}}\right)^{2}, \quad T_{1}(r) \sim \frac{1}{4} \ln A, \quad T_{3}(r) \sim \frac{1}{2} \ln B, \quad \phi^{i}(r) \sim \phi_{0}^{i},
$$

with $r_{\text {AdS }}$ the curvature radius of the $\mathrm{AdS}_{2}$ part, $A$ and $B$ positive constants and $\phi_{0}^{i}$ the horizon values of the scalar fields. The Einstein equations (3.14) become then algebraic and admit the solution

$$
A=-\frac{\Sigma_{0}}{g^{2} U_{0}}, \quad B=-\frac{2}{g^{2} U_{0}}, \quad r_{\text {AdS }}^{2}=-\frac{1}{g^{2} U_{0}},
$$

where $U_{0} \equiv U\left(\phi_{0}^{i}\right)<0$ and $\Sigma_{0} \equiv G_{I J}\left(\phi_{0}^{i}\right) p^{I} p^{J}$. Using (6.1) and (6.2), the equations (3.15) for the scalars boil down to

$$
\left.\partial_{i} V_{\mathrm{eff}}\right|_{\phi_{0}^{i}}=0
$$

where

$$
V_{\text {eff }}\left(\phi^{i}\right)=\frac{\sqrt{G_{I J}\left(\phi^{i}\right) p^{I} p^{J}}}{2 \sqrt{2} g^{2}\left|U\left(\phi^{i}\right)\right|}
$$

is an effective potential whose normalization has been chosen for later convenience. Thus, the attractor solution reads

$$
\begin{aligned}
& d s^{2}=-g^{2}\left|U_{0}\right| r^{2} d t^{2}+\frac{d r^{2}}{g^{2}\left|U_{0}\right| r^{2}}+\sqrt{\frac{\Sigma_{0}}{g^{2}\left|U_{0}\right|}}\left(e^{2 z} d x^{2}+e^{-2 z} d y^{2}\right)+\frac{2}{g^{2}\left|U_{0}\right|} d z^{2}, \\
& F^{I}=p^{I} d x \wedge d y, \quad \phi^{i}(r)=\phi_{0}^{i} .
\end{aligned}
$$

The horizon values $\phi_{0}^{i}$ of the scalars are computed by extremization of the effective potential (6.4) and (unless $V_{\text {eff }}$ has flat directions) are completely fixed by the magnetic charges and the constants $V_{I}$, in accordance with the attractor mechanism. Finally, the entropy density is given by

$$
s=V_{\mathrm{eff}}\left(\phi_{0}^{i}\right) .
$$

Notice that, even if $V_{\text {eff }}$ has flat directions, and thus (some of) the moduli at the horizon are not stabilized, (6.7) implies that the Bekenstein-Hawking entropy is given by the value of $V_{\text {eff }}$ at its minimum, which depends only on the magnetic charges $p^{I}$ and the parameters $V_{I}$. As a consequence of the results of section 5.2, the attractor geometry (6.5), (6.6) breaks all the supersymmetries.

As an example, we consider the stu model, which involves two vector multiplets, and has $C_{012}=1$ and its permutations as only nonvanishing components of $C_{I J K}$. We define $t=\phi^{1}, u=\phi^{2}$, and choose the parametrization $h^{1}=t, h^{2}=u$ and $h^{0}=s=(t u)^{-1}$, 
where the last relation follows from (2.3). Using the expresssions of section 2 and taking $V_{I}=1 / 3 \forall I$, we get

$$
\begin{aligned}
G_{I J} & =\frac{1}{2} \operatorname{diag}\left(s^{-2}, t^{-2}, u^{-2}\right), \quad \mathcal{G}_{i j}=\left[\begin{array}{cc}
\frac{1}{t^{2}} & \frac{1}{2 t u} \\
\frac{1}{2 t u} & \frac{1}{u^{2}}
\end{array}\right], \\
U(t, u) & =-2\left(t u+\frac{1}{t}+\frac{1}{u}\right) .
\end{aligned}
$$

The effective potential (6.4) becomes

$$
V_{\text {eff }}(t, u)=\frac{\sqrt{\left(p^{0}\right)^{2} t^{2} u^{2}+\left(p^{1}\right)^{2} t^{-2}+\left(p^{2}\right)^{2} u^{-2}}}{8 g^{2}\left(t u+t^{-1}+u^{-1}\right)},
$$

and the eqs. (6.3) boil down to

$$
\begin{aligned}
& \left(p^{0}\right)^{2} t^{3} u^{3}(t+2 u)-\left(p^{1}\right)^{2}\left(u+2 t u^{3}\right)-\left.\left(p^{2}\right)^{2}\left(t^{3} u-t\right)\right|_{t_{0}, u_{0}}=0 \\
& \left(p^{0}\right)^{2} t^{3} u^{3}(2 t+u)-\left(p^{1}\right)^{2}\left(t u^{3}-u\right)-\left.\left(p^{2}\right)^{2}\left(t+2 t^{3} u\right)\right|_{t_{0}, u_{0}}=0 .
\end{aligned}
$$

\subsection{Electric ansatz}

We now consider the case of purely electric field strengths. For a horizon modelled on solvegeometry, by means of the constraint (3.9) and the structure constants (A.6), the fourth eq. of (3.6) reduces to $(A=1,3)$

$$
\begin{aligned}
-V^{\prime} T_{1}^{\prime}-V T_{1}^{\prime \prime}-V T_{1}^{\prime}\left(2 T_{1}^{\prime}+T_{3}^{\prime}\right) & =\frac{1}{3} e^{-2\left(2 T_{1}+T_{3}\right)} G^{I J} q_{I} q_{J}+\frac{2}{3} g^{2} U, \\
-V^{\prime} T_{3}^{\prime}-V T_{3}^{\prime \prime}-V T_{3}^{\prime}\left(2 T_{1}^{\prime}+T_{3}^{\prime}\right)-2 e^{-2 T_{3}} & =\frac{1}{3} e^{-2\left(2 T_{1}+T_{3}\right)} G^{I J} q_{I} q_{J}+\frac{2}{3} g^{2} U,
\end{aligned}
$$

which immediately implies that a configuration with $T_{1}$ and $T_{3}$ constant is not acceptable. ${ }^{9}$ One can try to relax the ansatz on $T_{1}$ and $T_{3}$ by assuming a generic power dependence like

$$
e^{2 T_{1}} \sim k_{1} r^{\alpha_{1}}, \quad e^{2 T_{3}} \sim k_{3} r^{\alpha_{3}},
$$

with $k_{A}$ and $\alpha_{A}$ constants, but consistency of eqs. (6.12) requires $\alpha_{A}=0$ and we fall into the previous contradictory case.

For a horizon modelled on nilgeometry, cf. (A.9)-(A.11), the fourth eq. of (3.6) gives

$$
\begin{aligned}
& -V^{\prime} T_{1}^{\prime}-V T_{1}^{\prime \prime}-V T_{1}^{\prime} \sum_{B=1}^{3} T_{B}^{\prime}+\frac{1}{2} e^{2\left(T_{1}-T_{2}-T_{3}\right)}=\frac{q^{2}}{3} e^{-2\left(T_{1}+T_{2}+T_{3}\right)}+\frac{2}{3} g^{2} U \\
& -V^{\prime} T_{2}^{\prime}-V T_{2}^{\prime \prime}-V T_{2}^{\prime} \sum_{B=1}^{3} T_{B}^{\prime}-\frac{1}{2} e^{2\left(T_{1}-T_{2}-T_{3}\right)}=\frac{q^{2}}{3} e^{-2\left(T_{1}+T_{2}+T_{3}\right)}+\frac{2}{3} g^{2} U \\
& -V^{\prime} T_{3}^{\prime}-V T_{3}^{\prime \prime}-V T_{3}^{\prime} \sum_{B=1}^{3} T_{B}^{\prime}-\frac{1}{2} e^{2\left(T_{1}-T_{2}-T_{3}\right)}=\frac{q^{2}}{3} e^{-2\left(T_{1}+T_{2}+T_{3}\right)}+\frac{2}{3} g^{2} U
\end{aligned}
$$

\footnotetext{
${ }^{9}$ For $T_{1}^{\prime}=T_{3}^{\prime}=0$, the difference of the two eqs. leads to $e^{-2 T_{3}}=0$.
} 
where $q^{2}=G^{I J} q_{I} q_{J}$. Again, an ansatz with $T_{1}, T_{2}$ and $T_{3}$ constant does not work, since in that case the difference of the first and the second eq. of (6.14) yields

$$
e^{2\left(T_{1}-T_{2}-T_{3}\right)}=0 \text {. }
$$

If we assume $e^{2 T_{A}} \sim k_{A} r^{\alpha_{A}}$ and plug this ansatz into (6.14), we end up with $\alpha_{A}=0$, which we have just seen to lead to a contradiction. One obtains thus the following

Proposition 3. There are no static attractors with Sol or Nil horizons and purely electric field strengths.

\section{Acknowledgments}

This work was supported partly by INFN and by MIUR-PRIN contract 2017CC72MK003.

\section{A Homogeneous manifolds}

Let $M$ be a (pseudo)-Riemannian manifold with isometry group $G . \quad M$ is said to be homogeneous if $G$ acts transitively on $M$, i.e. if $\forall p, q \in M$ there exists an isometry $\phi \in G$ such that $\phi(p)=q$. The action of $G$ on $M$ is called simply transitive if the element $\phi$ is unique or, equivalently, if $\operatorname{dim} M=\operatorname{dim} G$. In this case, $M$ itself is said to be simply transitive.

Let us restrict our discussion to a simply transitive manifold. Since $\operatorname{dim} M=\operatorname{dim} G$, the Killing vectors $\xi_{A}(A=1, \ldots, \operatorname{dim} M)$ form a basis of the tangent space. However, it is more convenient [25] to choose a $G$-invariant basis $X_{A}$, i.e., a basis such that

$$
\mathcal{L}_{\xi_{B}} X_{A}=\left[\xi_{B}, X_{A}\right]=0 \quad \forall A, B,
$$

with $\mathcal{L}_{\xi_{B}} X_{A}$ the Lie derivative of the vector field $X_{A}$ along $\xi_{B}$. The dual basis $\theta^{A}$ of a $G$-invariant basis $X_{A}$ is also $G$-invariant, $\mathcal{L}_{\xi_{B}} \theta^{A}=0$, and satisfies

$$
d \theta^{A}=\frac{1}{2} C_{B C}^{A} \theta^{B} \wedge \theta^{C},
$$

with $C_{B C}^{A}$ the structure constants of the Lie algebra of $G$. Furthermore, a simply transitive homogeneous manifold can be equipped with a metric

$$
d s^{2}=g_{A B} \theta^{A} \theta^{B},
$$

where the components $g_{A B}$ are constant on $M$.

Bianchi showed that in total there are nine three-dimensional Lie algebras, the socalled nine Bianchi cosmologies, labelled from type I to type IX. The name 'cosmologies' comes from the fact that these manifolds are used as spatial sections in many spatially homogeneous but anisotropic cosmological models. The Bianchi cosmologies are divided into two classes, $\mathrm{A}$ and $\mathrm{B}$, according to the way the structure constants $C_{B C}^{A}$ can be expanded (see table 6.2 of [25] for details). In particular, class A spacetimes satisfy

$$
\sum_{A} C_{A B}^{A}=0 \text {. }
$$




\begin{tabular}{|l|l|}
\hline Bianchi & Thurston \\
\hline I, VII $_{0}$ & $\mathrm{E}^{3}$ \\
\hline II & Nil \\
\hline VI $_{-1}$ & Sol \\
\hline VIII & $\widetilde{\text { SL }}(2, \mathbb{R})$ \\
\hline IX & $\mathrm{S}^{3}$ \\
\hline
\end{tabular}

\begin{tabular}{|l|l|}
\hline Bianchi & Thurston \\
\hline III & $\mathrm{H}^{2} \times \mathbb{R}$ \\
\hline $\mathrm{V}, \mathrm{VII}_{h \neq 0}$ & $\mathrm{H}^{3}$ \\
\hline
\end{tabular}

Table 1. Class A (left) and B (right) spacetimes and corresponding Thurston geometries.

An important result in geometric topology is the Thurston conjecture [44], which states that every three-dimensional closed and orientable manifold has a geometric structure modelled on one of the eight model geometries

$$
\mathrm{S}^{3}, \quad \mathrm{E}^{3}, \quad \mathrm{H}^{3}, \quad \mathrm{~S}^{2} \times \mathbb{R}, \quad \mathrm{H}^{2} \times \mathbb{R}, \quad \text { Nil }, \quad \text { Sol }, \quad \widetilde{\mathrm{SL}}(2, \mathbb{R}),
$$

where $\widetilde{\mathrm{SL}}(2, \mathbb{R})$ is the universal covering of $\mathrm{SL}(2, \mathbb{R})$. In $[45]$ it was shown that there exists a correspondence, not necessarily one to one, between the nine Bianchi cosmologies and the eight Thurston model geometries, which is summarized in table $1 .^{10}$ In the following, we list explicitely the metrics for solvegeometry/VI -1 and nilgeometry/II in terms of $G$-invariant one-forms $\theta^{A}$, as well as the nonvanishing structure constants of the related Lie algebras.

- Solvegeometry:

$$
\begin{array}{rlrl}
C_{13}^{1} & =-C_{31}^{1}=1, \quad C_{23}^{2}=-C_{32}^{2}=-1, & \\
\theta^{1} & =e^{z} d x, & \theta^{2}=e^{-z} d y, & \theta^{3}=-d z, \\
d s^{2} & =e^{2 z} d x^{2}+e^{-2 z} d y^{2}+d z^{2} .
\end{array}
$$

- Nilgeometry:

$$
\begin{aligned}
C_{23}^{1} & =-C_{32}^{1}=1, \\
\theta^{1} & =d z-x d y, \quad \theta^{2}=d y, \quad \theta^{3}=d x, \\
d s^{2} & =(d z-x d y)^{2}+d y^{2}+d x^{2} .
\end{aligned}
$$

Open Access. This article is distributed under the terms of the Creative Commons Attribution License (CC-BY 4.0), which permits any use, distribution and reproduction in any medium, provided the original author(s) and source are credited.

\footnotetext{
${ }^{10}$ The Bianchi types IV and $\mathrm{VI}_{h \neq-1}$ are not contained in this correspondence. Moreover, the Thurston geometry $\mathrm{S}^{2} \times \mathbb{R}$ is missing since it corresponds to the Kantowski-Sachs model, in which $G$ does not act simply transitively or does not possess a subgroup with simply transitive action.
} 


\section{References}

[1] S.W. Hawking, Black holes in general relativity, Commun. Math. Phys. 25 (1972) 152 [INSPIRE].

[2] S.W. Hawking and G.F.R. Ellis, The large scale structure of space-time, Cambridge University Press, Cambridge, U.K. (1973).

[3] S.W. Hawking, The event horizon, in Black Holes, Les Houches Lectures, C. DeWitt and B.S. DeWitt eds., North Holland, Amsterdam, The Netherlands (1972).

[4] R. Emparan and H.S. Reall, A rotating black ring solution in five-dimensions, Phys. Rev. Lett. 88 (2002) 101101 [hep-th/0110260] [INSPIRE].

[5] G.J. Galloway and R. Schoen, A generalization of Hawking's black hole topology theorem to higher dimensions, Commun. Math. Phys. 266 (2006) 571 [gr-qc/0509107] [INSPIRE].

[6] J.P.S. Lemos, Cylindrical black hole in general relativity, Phys. Lett. B 353 (1995) 46 [gr-qc/9404041] [INSPIRE].

[7] R.B. Mann, Pair production of topological anti-de Sitter black holes, Class. Quant. Grav. 14 (1997) L109 [gr-qc/9607071] [INSPIRE].

[8] L. Vanzo, Black holes with unusual topology, Phys. Rev. D 56 (1997) 6475 [gr-qc/9705004] [INSPIRE].

[9] R.-G. Cai and Y.-Z. Zhang, Black plane solutions in four-dimensional space-times, Phys. Rev. D 54 (1996) 4891 [gr-qc/9609065] [INSPIRE].

[10] A. Gnecchi, K. Hristov, D. Klemm, C. Toldo and O. Vaughan, Rotating black holes in $4 d$ gauged supergravity, JHEP 01 (2014) 127 [arXiv:1311.1795] [INSPIRE].

[11] D. Klemm, Four-dimensional black holes with unusual horizons, Phys. Rev. D 89 (2014) 084007 [arXiv: 1401.3107] [INSPIRE].

[12] R.A. Hennigar, D. Kubizñák and R.B. Mann, Entropy Inequality Violations from Ultraspinning Black Holes, Phys. Rev. Lett. 115 (2015) 031101 [arXiv:1411.4309] [InSPIRE].

[13] D. Birmingham, Topological black holes in Anti-de Sitter space, Class. Quant. Grav. 16 (1999) 1197 [hep-th/9808032] [INSPIRE].

[14] C. Cadeau and E. Woolgar, New five-dimensional black holes classified by horizon geometry and a Bianchi VI brane world, Class. Quant. Grav. 18 (2001) 527 [gr-qc/0011029] [INSPIRE].

[15] R.E. Arias and I. Salazar Landea, Thermoelectric Transport Coefficients from Charged Solv and Nil Black Holes, JHEP 12 (2017) 087 [arXiv: 1708.04335] [INSPIRE].

[16] M. Bravo-Gaete and M. Hassaïne, Thermodynamics of dyonic black holes with Thurston horizon geometries, Phys. Rev. D 97 (2018) 024020 [arXiv:1710.02720] [INSPIRE].

[17] S. Ferrara, R. Kallosh and A. Strominger, $N=2$ extremal black holes, Phys. Rev. D 52 (1995) R5412 [hep-th/9508072] [INSPIRE].

[18] A. Strominger, Macroscopic entropy of $N=2$ extremal black holes, Phys. Lett. B 383 (1996) 39 [hep-th/9602111] [INSPIRE].

[19] S. Ferrara and R. Kallosh, Supersymmetry and attractors, Phys. Rev. D 54 (1996) 1514 [hep-th/9602136] [INSPIRE]. 
[20] S. Ferrara and R. Kallosh, Universality of supersymmetric attractors, Phys. Rev. D 54 (1996) 1525 [hep-th/9603090] [INSPIRE].

[21] S. Ferrara, G.W. Gibbons and R. Kallosh, Black holes and critical points in moduli space, Nucl. Phys. B 500 (1997) 75 [hep-th/9702103] [INSPIRE].

[22] N. Iizuka, S. Kachru, N. Kundu, P. Narayan, N. Sircar and S.P. Trivedi, Bianchi Attractors: A Classification of Extremal Black Brane Geometries, JHEP 07 (2012) 193 [arXiv: 1201.4861] [INSPIRE].

[23] S. Kachru, N. Kundu, A. Saha, R. Samanta and S.P. Trivedi, Interpolating from Bianchi Attractors to Lifshitz and AdS Spacetimes, JHEP 03 (2014) 074 [arXiv:1310.5740] [INSPIRE].

[24] M. Günaydin, G. Sierra and P.K. Townsend, Gauging the $d=5$ Maxwell-Einstein Supergravity Theories: More on Jordan Algebras, Nucl. Phys. B 253 (1985) 573 [InSPIRE].

[25] M.P. Ryan and L.C. Shepley, Homogeneous relativistic cosmologies, Princeton Series In Physics, Princeton University Press, Princeton, Usa, (1975).

[26] A. Ashtekar and A. Magnon, Asymptotically anti-de Sitter space-times, Class. Quant. Grav. 1 (1984) L39 [INSPIRE].

[27] A. Ashtekar and S. Das, Asymptotically Anti-de Sitter space-times: Conserved quantities, Class. Quant. Grav. 17 (2000) L17 [hep-th/9911230] [INSPIRE].

[28] J.B. Gutowski and H.S. Reall, General supersymmetric AdS $S_{5}$ black holes, JHEP 04 (2004) 048 [hep-th/0401129] [INSPIRE].

[29] D. Klemm and W.A. Sabra, Supersymmetry of black strings in $D=5$ gauged supergravities, Phys. Rev. D 62 (2000) 024003 [hep-th/0001131] [INSPIRE].

[30] J.B. Gutowski and H.S. Reall, Supersymmetric AdS $S_{5}$ black holes, JHEP 02 (2004) 006 [hep-th/0401042] [INSPIRE].

[31] U. Kayani, Symmetry enhancement of extremal horizons in $D=5$ supergravity, Class. Quant. Grav. 35 (2018) 125013 [arXiv:1801.08833] [INSPIRE].

[32] J. Grover, J.B. Gutowski, G. Papadopoulos and W.A. Sabra, Index Theory and Supersymmetry of 5D Horizons, JHEP 06 (2014) 020 [arXiv: 1303.0853] [INSPIRE].

[33] K. Goldstein, N. Iizuka, R.P. Jena and S.P. Trivedi, Non-supersymmetric attractors, Phys. Rev. D 72 (2005) 124021 [hep-th/0507096] [INSPIRE].

[34] D. Astefanesei, K. Goldstein, R.P. Jena, A. Sen and S.P. Trivedi, Rotating attractors, JHEP 10 (2006) 058 [hep-th/0606244] [INSPIRE].

[35] J.F. Morales and H. Samtleben, Entropy function and attractors for AdS black holes, JHEP 10 (2006) 074 [hep-th/0608044] [INSPIRE].

[36] M. Huebscher, P. Meessen, T. Ortín and S. Vaulà, Supersymmetric $N=2$ Einstein-Yang-Mills monopoles and covariant attractors, Phys. Rev. D 78 (2008) 065031 [arXiv: 0712.1530] [INSPIRE].

[37] S. Bellucci, S. Ferrara, A. Marrani and A. Yeranyan, $d=4$ Black Hole Attractors in $N=2$ Supergravity with Fayet-Iliopoulos Terms, Phys. Rev. D 77 (2008) 085027 [arXiv: 0802.0141] [INSPIRE].

[38] S.L. Cacciatori and D. Klemm, Supersymmetric AdS 4 black holes and attractors, JHEP 01 (2010) 085 [arXiv:0911.4926] [INSPIRE]. 
[39] G. Dall'Agata and A. Gnecchi, Flow equations and attractors for black holes in $N=2 \mathrm{U}(1)$ gauged supergravity, JHEP 03 (2011) 037 [arXiv: 1012.3756] [INSPIRE].

[40] S. Kachru, R. Kallosh and M. Shmakova, Generalized Attractor Points in Gauged Supergravity, Phys. Rev. D 84 (2011) 046003 [arXiv:1104.2884] [InSPIRE].

[41] S. Chimento, D. Klemm and N. Petri, Supersymmetric black holes and attractors in gauged supergravity with hypermultiplets, JHEP 06 (2015) 150 [arXiv: 1503.09055] [INSPIRE].

[42] P. Meessen and T. Ortín, Non-Extremal Black Holes of $N=2, d=5$ Supergravity, Phys. Lett. B 707 (2012) 178 [arXiv:1107.5454] [INSPIRE].

[43] A. de Antonio Martín, T. Ortín and C.S. Shahbazi, The FGK formalism for black p-branes in d dimensions, JHEP 05 (2012) 045 [arXiv: 1203.0260] [INSPIRE].

[44] W.P. Thurston, Three-dimensional geometry and topology, S. Levy ed., Princeton University Press, Princeton, U.S.A. (1997).

[45] H.V. Fagundes, Closed spaces in cosmology, Gen. Rel. Grav. 24 (1992) 199 [Gen. Rel. Grav. 30 (1998) 1437] [arXiv:0812.4103] [INSPIRE]. 